\title{
Anthropomorphism Indexes of the Kinematic Chain for Artificial Hands
}

\author{
Immaculada Llop-Harillo*, Antonio Pérez-González, Javier Andrés-Esperanza \\ Grupo de Biomecánica y Ergonomía, Departamento de Ingeniería Mecánica y Construcción, Universitat Jaume I (UJI), \\ Castellón 12071, Spain
}

\begin{abstract}
Anthropomorphic hands have received increasing research interest in the fields of robotics and prosthetics. But it is not yet clear how to evaluate their anthropomorphism. Similarity in the kinematic chain is essential to achieve both functionality and cosmesis. A few previous works have addressed the definition of anthropomorphism indexes, although they have some limitations in its definition. In this study, three different anthropomorphism indexes have been defined to compare the kinematic chain of artificial hands with that of the human hand. These indexes are based on the comparison of: (1) the parameters of the kinematic chain (dimensions, type of joints, orientations and ranges of motion), (2) the reachable workspace, and (3) common grasping postures. Five artificial hands with different degrees of anthropomorphism have been compared using the three Anthropomorphism Indexes of the Kinematic Chain (AIKC). The results show a high correlation between the first and third AIKC for the hands compared. The second AIKC presents much lower values than the other two, although they are higher for hands that combine abduction/adduction and flexion/extension movements in the kinematic chain of each finger. These indexes can be useful during the initial stage of designing artificial hands or evaluating their anthropomorphism.
\end{abstract}

Keywords: anthropomorphism, grasping, indexes, kinematics, multifingered hands

Copyright $(C)$ The author(s) 2020.

\section{Introduction}

Research interest in anthropomorphic hands has increased recently, fostered by the needs imposed by humanoid robotics and human-robot cooperation ${ }^{[1-3]}$ and also by hand prosthetics ${ }^{[4]}$. The popularization of additive manufacturing has also boosted the number of low-cost proposals for prosthetic applications ${ }^{[5]}$, promoted by initiatives such as e-NABLE (http://enablingthefuture.org/), the Open Hand Project ${ }^{[6]}$ and Open Bionics ${ }^{[7]}$. However, according to several studies ${ }^{[8-11]}$, the degree of functionality of the Human Hand $(\mathrm{HH})$ is far from being achieved even by expensive commercial prostheses, such as i-Limb ${ }^{[12]}$, or sophisticated robotic hands such as Shadow Dexterous Hand ${ }^{[13]}$.

At the same time, there are no methods to assess this anthropomorphism and even an agreement on the meaning of this concept is lacking. Anthropomorphism can be understood as similarity to the $\mathrm{HH}$ in terms of size, weight, shape, appearance, temperature, etc., i.e. $\operatorname{cosmesis}^{[5]}$, or as similarity in terms of functionality or dexterity $^{[3]}$. The Kinematic Chain (KC) of the Artificial
Hand (AH) is of primary importance to achieve anthropomorphism in both senses: cosmesis and functionality. The whole $\mathrm{KC}$ of a hand is composed of as many open KCs as there are fingers. The length of the finger segments or links and the position and orientation of their joints determine the postures that the hand can adopt, or in robotic terminology, the reachable workspace $^{[14]}$. The maximization of the degree of anthropomorphism of the $\mathrm{KC}$ of an $\mathrm{AH}$ during the design process depends, primarily, on having adequate metrics or indexes that allow its quantification.

Some previous works have attempted to quantify hand anthropomorphism. In Ref. [15], a method for calculating an Anthropomorphism Index (AI) was proposed, based on weighting the qualitative evaluation of three aspects of the $\mathrm{AH}$ in comparison to the $\mathrm{HH}$ : the $\mathrm{KC}$, the contact surfaces, and the dimensions. This method was used, together with a potential dexterity index considering the control system and sensors, to compare different anthropomorphic hands. The main limitation of this approach is the dependence on qualitative assessments. A similar approach was proposed in Ref. [16]

\footnotetext{
*Corresponding author: Immaculada Llop-Harillo

E-mail: illop@uji.es
} 
based on the evaluation of twelve aspects related to mechanics, rehabilitation and anatomy. The attainable grasp gestures and the distribution of the rotation axes were considered relevant aspects for anthropomorphism in that study. Recently, the authors also defined an Anthropomorphism Index of Mobility (AIM) ${ }^{[17]}$ based on the topology of the whole hand, joints and Degrees of Freedom (DoFs), and the possibility of controlling these DoFs independently (number of actuators and type of underactuation). The weighting factors to compute the AIM depend on the relevance of the different groups of DoFs of the hand according to a human grasp experiment on the most important grasp types. The computation of AIM is straightforward and useful in the concept design stage, but it does not consider some aspects like the orientation of the joint axes, the range of motion of the joints or the dimension of the phalanges. Feix et al.$^{[18]}$ proposed an AI based on comparing the space of positions and orientations attainable by the fingertips of the $\mathrm{AH}$ and $\mathrm{HH}$. Given the high number of parameters involved in the comparison, they used dimensional reduction techniques to define the index. Despite pioneering the methods for comparing $\mathrm{KC}$ among hands, it has the limitation of considering only the fingertips to define the workspace of the hand. In a similar approach, Liarokapis et al.$^{[19]}$ included more points of the hand in the comparison and also the orientations of the finger base frame. However, they used convex-hulls to compare the workspaces and Euler angles to compare the orientations, which can introduce some errors in the comparison, as explained later in this paper.

The aim of the present study is to define and compare new indexes to measure the anthropomorphism of the $\mathrm{KC}$ of an $\mathrm{AH}$, while attempting to solve some of the limitations observed in AIs defined in the literature. The new indexes are intended to be quantitative and calculable from the basic information about the KC. They will be defined to range from 0 (low anthropomorphism) to 1 (HH, or a perfect mimicry of it). The KC will be compared here in terms of the length of the links and the position, orientation, and mobility of the joints.

\section{Kinematic chain description}

\subsection{Kinematic chain of the human hand}

The KC of each digit of the HH (Fig. 1) is com- posed of four segments, in proximal to distal order: metacarpal (MC), Proximal Phalanx (PP), Medial Phalanx (MP) and Distal Phalanx (DP), except for the thumb, which lacks the MP. The MCs of the long fingers constitute the palm of the hand and have scarce mobility between them, although the carpometacarpal joints (CMC) have increasing mobility from the index to the little finger, thereby providing the palmar $\operatorname{arching}^{[20]}$. The CMC joint of the thumb has the highest mobility, with two rotational DoFs around two nearly perpendicular axes, which enable the opposition of the thumb to the long fingers ${ }^{[20,21]}$. The metacarpophalangeal joints (MCP) also have two rotational DoFs with nearly perpendicular axes: flexion/extension $(\mathrm{F} / \mathrm{E})$ in the sagittal plane and abduction/adduction $(\mathrm{Ab} / \mathrm{Ad})$ in the frontal plane $^{[20]}$. Finally, the proximal interphalangeal joints (PIP), the distal interphalangeal joints (DIP) of the long fingers, and the interphalangeal joint (IP) of the thumb have one DoF in the sagittal plane that enables their F/E. Altogether, the $\mathrm{KC}$ of the hand exhibits $25 \mathrm{DoFs}$, five per finger, or 23 DoFs if the mobility of the CMC joints of the index and middle fingers is neglected, as in some previous models ${ }^{[22]}$.

In this work, we assume a fixed position of the rotational axes with respect to the bones. Moreover, the

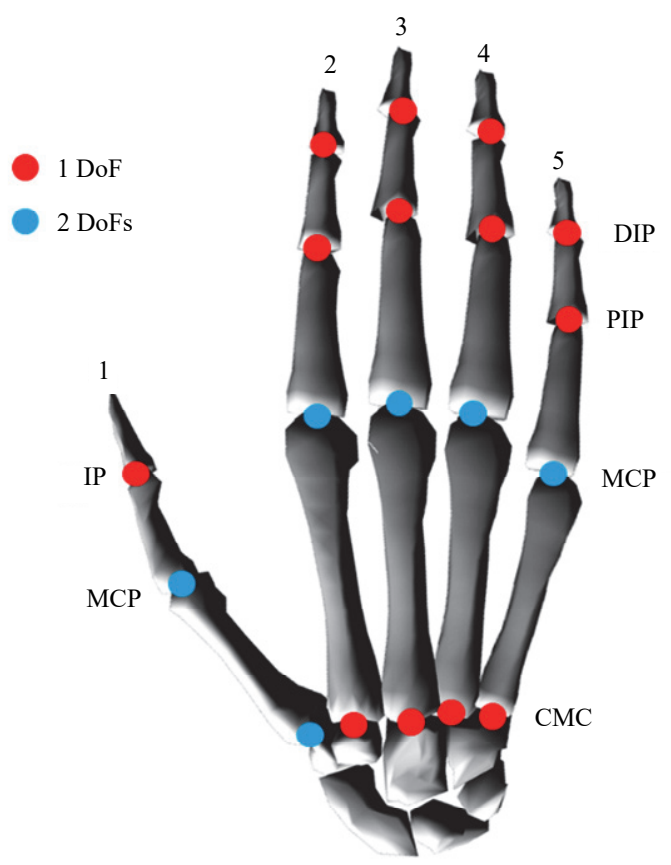

Fig. 1 Kinematic chain of the human hand. The abbreviations of the joints and their DoFs are indicated. 
joints with two DoFs are considered as universal joints (perpendicular axes that intersect), although according to some studies, the axes of the joints with two DoFs are neither exactly perpendicular to each other nor do they intersect at the same point, with variations from one person to another ${ }^{[21]}$. These simplifications are common for most biomechanical models of the hand ${ }^{[22-25]}$ and imply a small error in the reachable positions.

\subsection{Definition of the kinematic chain}

In order to define the $\mathrm{KC}$ of a hand, some simplifications are considered: the links are rigid and their transversal dimensions are neglected; joint mobility is independent of the finger posture; and motion coupling among joints imposed by the actuation systems is obviated. A Local Coordinate System (LCS) is associated with each segment (bone in the case of the $\mathrm{HH}$ ) and the position and orientation of the joints are related to these LCSs. In robotics, the Denavit-Hartenberg (DH) convention $^{[26]}$ is commonly used to define the $\mathrm{KC}$ of a serial robot. It allows the $\mathrm{KC}$ to be defined with a minimum of four parameters for each segment of the chain: two rotations and two translations. However, the convention is ambiguous if the precise way of locating the positive direction of the axes is not defined and presents more than one possible solution in the case of parallel axes. Additionally, the use of this convention in the case of consecutive joints with coplanar and almost parallel rotation axes causes the location of the origin of each
LCS not to match the center of the real joints. For example, in the $\mathrm{HH}$, if we assume the flexion axis of the wrist and the flexion axis of the CMC joint of the little finger as two consecutive $Z$-axes of the serial chain, the DH convention will locate the LCS of the latter far from the metacarpal bone axis. In sum, although the DH convention may define the chain with a minimum number of parameters, it has the disadvantage that some of them are not very intuitive from an anatomical or topological point of view.

Therefore, in this study we propose to locate the LCS for each segment of the KC in the center of the joint with the previous segment. Fig. 2 shows the orientation of the LCSs associated with the $\mathrm{KC}$ of the index finger and thumb for a right hand. Specifically, those LCSs were defined with the following criteria: $Z$-axis coincident with the $\mathrm{F} / \mathrm{E}$ axis of the joint, and directed so that flexion corresponds to a positive rotation around $Z$; $X$-axis pointing in the palmar-dorsal direction, thus indicating the $\mathrm{Ab} / \mathrm{Ad}$ axis of the joint; and $Y$-axis defining a right-handed trihedron, pointing towards the distal end of the fingers. In this way, an LCS exhibits positive $Y$-values with respect to another preceding (more proximal) LCS. This criterion is similar to that proposed by the International Society of Biomechanics ${ }^{[27]}$, with the difference that $X$ and $Y$ axes have opposite directions.

The fixed LCS for all the digits is located at the center of the wrist, see $\left\{L C S_{0}\right\}$ in Fig. 2. The Reference Posture (RP) of the hand is defined as that, within the

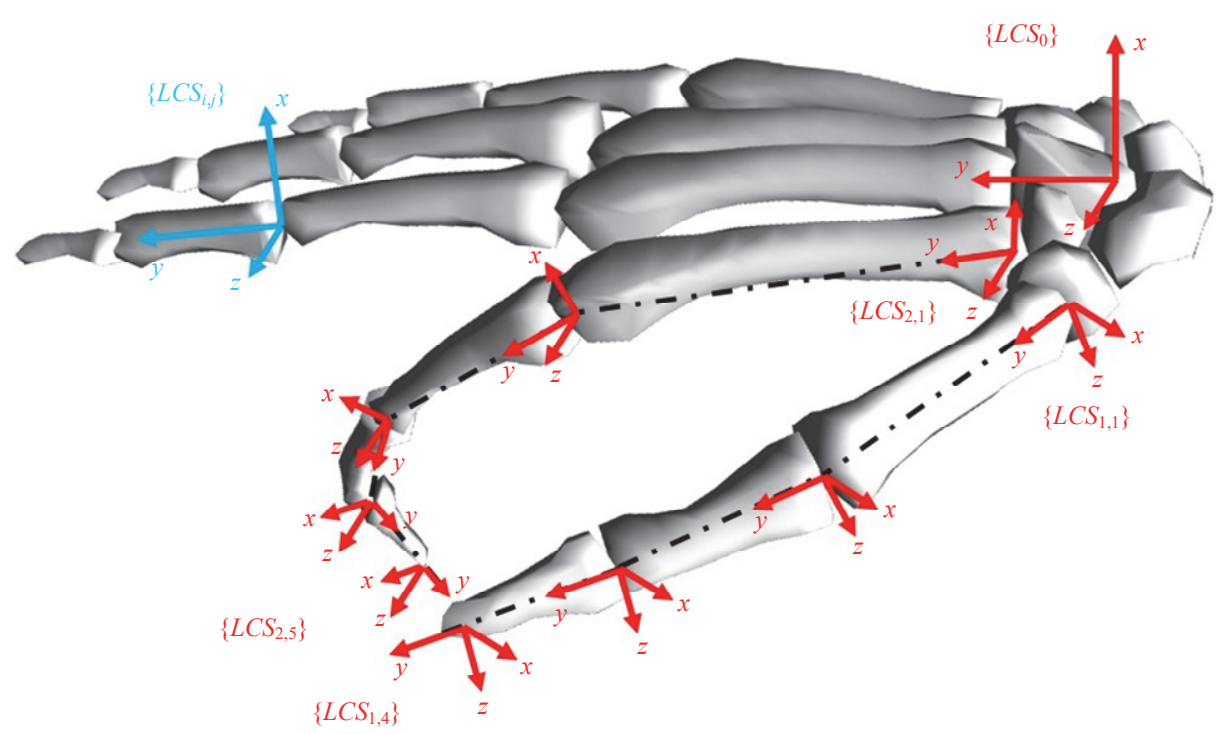

Fig. 2 Proposal for the location of the $L C S_{i, j}$ in each significant point $(j)$ of each digit $(i)$ of the hand. 
range of mobility of the hand, corresponding to the smallest possible absolute $X$ coordinate (expressed in $\left\{L C S_{0}\right\}$ ) for the origin of every LCS. The KC of each digit is defined by means of three displacements and three rotations of each LCS with respect to its immediate proximal in the chain in the RP. Specifically, the LCS of segment $j$ (1: MC, 2: PP, 3: MP, 4: DP, 5: Fingertip) of digit $i$ (1: thumb, 2: index, 3: middle, 4 : ring, 5 : little) is defined with a six-element vector:

$$
\begin{aligned}
& \boldsymbol{v}_{i, j}=\left[\begin{array}{llllll}
x_{i, j} & y_{i, j} & z_{i, j} & \theta_{x_{i, j}} & \theta_{y_{i, j}} & \theta_{z_{i, j}}
\end{array}\right], \\
& i=1,2, \ldots, 5 ; j=1,2, \ldots, 5,
\end{aligned}
$$

where the first three elements of the vector correspond to the translation of $L C S_{i, j}$ with respect to $L C S_{i, j-1}$ and the last three correspond to the Euler angles necessary to orient $\mathrm{LCS}_{i, j-1}$ as $\mathrm{LCS}_{i, j}$, with the order of rotations $X Z Y$. For convenience, one additional LCS is associated to each fingertip $(j=5)$; see Fig. 2. For digits with four segments (such as the thumb of the HH), the MP is considered inexistent, the $L C S_{i, 3}$ corresponds to the DP and the $L C S_{i, 4}$ corresponds to the fingertip.

The homogeneous transformation matrix between two consecutive LCSs $(j-1$ and $j$ ) in a digit $i$ can be easily obtained from Eq. (1) in the RP. For a different posture $(p)$, this transformation (Eq. (2)) will also be affected by the angles rotated in the joint between the two segments $\left(\beta_{i j} \mathrm{~F} / \mathrm{E}, \alpha_{i j} \mathrm{Ab} / \mathrm{Ad}\right)$ :

$$
\boldsymbol{T}_{i, j}^{p}=\left[\begin{array}{cc}
\boldsymbol{R}_{i, j}^{p} & \boldsymbol{t}_{i, j} \\
0 & 1
\end{array}\right],
$$

where

$$
\begin{gathered}
\boldsymbol{R}_{i, j}^{p}=\boldsymbol{R}_{x}\left(\theta_{x_{i, j}}\right) \cdot \boldsymbol{R}_{z}\left(\theta_{z_{i, j}}\right) \cdot \boldsymbol{R}_{y}\left(\theta_{y_{i, j}}\right) \cdot \boldsymbol{R}_{x}\left(\alpha_{i j}\right) \cdot \boldsymbol{R}_{z}\left(\beta_{i j}\right), \\
\boldsymbol{t}_{i, j}=\left[\begin{array}{l}
\left.x_{i, j} y_{i, j} z_{i, j}\right]^{\mathrm{T}}, \\
\boldsymbol{R}_{x}(\theta)=\left[\begin{array}{ccc}
1 & 0 & 0 \\
0 & \cos \theta & -\sin \theta \\
0 & \sin \theta & \cos \theta
\end{array}\right] \\
\boldsymbol{R}_{y}(\theta)=\left[\begin{array}{ccc}
\cos \theta & 0 & \sin \theta \\
0 & 1 & 0 \\
-\sin \theta & 0 & \cos \theta
\end{array}\right] \\
\boldsymbol{R}_{z}(\theta)=\left[\begin{array}{ccc}
\cos \theta & -\sin \theta & 0 \\
\sin \theta & \cos \theta & 0 \\
0 & 0 & 1
\end{array}\right]
\end{array}\right.
\end{gathered}
$$

The absolute position of the $L C S_{i, j}$, associated to segment $j$ of digit $i$, with respect to the fixed system $\left\{L C S_{0}\right\}$ located on the wrist, is obtained by successive multiplication of the transformation matrices of the consecutive segments:

$$
\boldsymbol{T}_{o_{-} i, j}^{p}=\boldsymbol{T}_{i, 1}^{p} \cdot \ldots \cdot \boldsymbol{T}_{i, j}^{p} .
$$

\section{Anthropomorphism Index of the Kinematic Chain (AIKC)}

To define an AIKC it is necessary to compare the $\mathrm{KC}$ of the $\mathrm{AH}$ with that of the $\mathrm{HH}$. The features involved in this comparison are the dimensions and the topology of the KC. The latter includes not only the type of joints and their orientation, but also their ranges of motion. These features determine the postures that the hand can achieve, and therefore influence its grasping ability and manipulability. Different alternatives to define this index are proposed below. In all of them the index is defined so that it ranges from 0 (low anthropomorphism) to $1(\mathrm{HH}$, or a perfect mimicry of it).

\section{1 $A I K C_{1}$ : based on the parameters of the kinematic chain}

A first strategy for comparing hands consists in defining a vector of characteristic parameters of the kinematic structure of the $\mathrm{AH}$ and comparing it with its corresponding vector of the HH. For each segment of the hand it is possible to define three characteristic vectors: one with the information about the relative position of the LCS of the segment with respect to its proximal segment (defined by Eq. (4)); a second vector with the orientation of the LCS with respect to its proximal segment (defined by the three Euler angles from Eq. (1)); and a third vector defining the limits of the rotation angles, $\beta_{i j}$ for $\mathrm{F} / \mathrm{E}$ and $\alpha_{i j}$ for $\mathrm{Ab} / \mathrm{Ad}$, that are allowed in the joints around the RP. The overall difference between these vectors in the human and the artificial models could be considered an error in the achievement of anthropomorphism. This error can be used to define a metric for the anthropomorphism of the KC.

The normalized error in the translation vectors between the $\mathrm{AH}$ and $\mathrm{HH}$ in each segment can be defined (Eq. (7)) as the Euclidean norm of the difference between the translation vector defined in Eq. (4) for the AH 
and its corresponding one for the $\mathrm{HH}, \boldsymbol{t}_{h_{i, j}}$, divided by a reference value for the lengths, $L_{\text {ref }}$. The length of the longest segment of the HH (MC bone of the index) has been taken as the reference in this study. This error value is limited to a maximum of 1 .

$$
e_{t_{i, j}}=\min \left(1,\left\|\boldsymbol{t}_{i, j}-\boldsymbol{t}_{h_{i, j}}\right\| / L_{\mathrm{ref}}\right) \text {. }
$$

The error in the orientation of the LCS requires a deeper analysis, as it is not correct to obtain it from the Euclidean norm of the difference between vectors containing Euler angles ${ }^{[28]}$. According to Ref. [28], the use of quaternions is the most correct and computationally efficient method to compare 3D rotations. Any rotation of a solid can be expressed as a rotation of angle $\theta$ around a unit vector $u_{x} \boldsymbol{i}+u_{y} \boldsymbol{j}+u_{z} \boldsymbol{k}$, and it is possible to express this rotation through the unit quaternion:

$$
\boldsymbol{q}=\cos \frac{\theta}{2}+\left(u_{x} \boldsymbol{i}+u_{y} \boldsymbol{j}+u_{z} \boldsymbol{k}\right) \sin \frac{\theta}{2} .
$$

A scalar product between two unit quaternions measures the difference between the two orientations ${ }^{[28]}$ and has the advantage of giving a result in the range $0-1$, where 1 corresponds to two significantly different orientations and 0 corresponds to two identical orientations. Therefore, the orientation error in each segment is defined as:

$$
e_{o_{i, j}}=1-\left|\boldsymbol{q}_{i, j} \cdot \boldsymbol{q}_{h_{i, j}}\right|,
$$

where $\boldsymbol{q}_{i, j}$ is the quaternion corresponding to the relative orientation between the $L C S_{i, j}$ and its preceding $L C S_{i, j-1}$ in the analyzed hand, and $\boldsymbol{q}_{h_{i, j}}$ is that of the HH. These quaternions have been obtained from the rotation matrix in the RP using the Matlab function rotm2quat. By default this function always selects the quaternion with the positive scalar part between the two opposites that represent the same rotation.

Finally, the error in the mobility range of each joint $j$ for digit $i$ is obtained by averaging the normalized absolute value of the difference between the limit ranges of the $\mathrm{AH}$ and $\mathrm{HH}$ :

$$
e_{r_{i, j}}=\frac{1}{2}\left[\min \left(1, \frac{\left|r_{i, j}-r_{h_{i, j}}\right|^{+}}{\pi / 2}\right)+\min \left(1, \frac{\left|r_{i, j}-r_{h_{i, j}}\right|^{-}}{\pi / 2}\right)\right],
$$

where $r_{i, j}$ is the mobility range limit of joint $j$ of digit $i$ of the $\mathrm{AH}$ and $r_{h_{i, j}}$ is that of the $\mathrm{HH}$, and the two addends correspond to the positive motion (flexion, abduction) around the RP and the negative one (extension, adduction). In order to normalize the error to a value between 0 and 1 , it is divided by $\pi / 2$ as a characteristic angle of the approximate maximum range of the hand flexion joints, limiting the maximum value of the error to 1 .

Using the translation, orientation and range error values obtained with Eqs. (7), (9) and (10), the $A I K C_{1}$ of a hand is defined as:

$$
\begin{aligned}
& A I K C_{1}=1- \\
& \quad \sum_{i=1}^{5} w_{i} \cdot\left[\frac{1}{n_{i}} \sum_{j=1}^{n_{i}} e_{t_{i, j}}+\frac{1}{n_{i}} \sum_{j=1}^{n_{i}} e_{e_{i, j}}+\frac{1}{m_{i}} \sum_{j=1}^{m_{i}} e_{r_{i, j}}\right] / 3,
\end{aligned}
$$

where $n_{i}$ is the number of segments of digit $i$ (4 for the thumb, and 5 for the long fingers), $m_{i}$ is the number of DoFs of digit $i$ ( 5 for all the digits), and the coefficient $w_{i}$ allows a different weighting of the error in each digit. Specifically in this study, it is proposed to use $w_{1}=0.5$ for the thumb and $w_{2}=w_{3}=w_{4}=w_{5}=0.125$ for each long finger, due to the predominant weight of the thumb in manipulation ${ }^{[29]}$. In cases where an $\mathrm{AH}$ has fewer fingers or segments than the $\mathrm{HH}$, a value of 1 should be assigned to the error associated with the missing segment in the preceding equations.

\section{2 $A I K C_{2}$ : based on the workspace}

The change in the length of the segments, the orientations of the axes or the range of mobility of the joints causes changes in the reachable workspace or, in other words, in the postures that the hand can adopt. It is possible to base the comparison of the $\mathrm{KC}$ on this workspace. Some previous works have used this strategy ${ }^{[18,19,30]}$, but with the limitations of including only the position of the fingertips ${ }^{[18]}$ or considering the differences in orientation only in the MCP joints ${ }^{[19]}$.

The space of the positions reached by a certain point of the hand can be obtained by moving all the DoFs of the KC of the fingers within their range of mobility. One way to quantify this workspace is to use the concepts of convex-hull ${ }^{[31]}$ or alpha-shape ${ }^{[32]}$. Both concepts attempt to quantify the space covered by a point cloud. To generate the alpha-shape, a spherical tool with radius alpha is moved to try to penetrate the cloud of points, which represents constraints on the movement 
of the tool. The space delimited by the points that impede the pass of the tool is the alpha-shape. The convex-hull is a particular case where the radius of the tool is infinite.

It is possible to compare hands by comparing the alpha-shape or convex-hull of the cloud of positions reachable by the tip and the centers of the joints of the digits. In this study, the use of the alpha-shape is proposed, as the convex-hull may include interior regions or other areas that do not strictly pertain to the reachable workspace. Before obtaining the alpha-shape, it is necessary to generate the space of reachable positions by moving all the DoFs of each finger within their range of mobility. In order to limit the magnitude of the problem to a reasonable number of points, we decided to generate random hand positions, moving each DoF simultaneously within its range of mobility. Through preliminary analyses, we observed that $n_{p}=10^{5}$ random postures covered the workspace reachable by the hand reasonably well, the error obtained in the value of the AI being lower than $0.1 \%$.

For the computation of $A I K C_{2}$, the following procedure was used:

(1) Computation of the 3D alpha-shapes of the point cloud corresponding to the origin of each $L C S_{i, j}$.

(2) Computation of $n_{i, j}$ as the number of points of the workspace generated by each $L C S_{i, j}$ of the HH that also lay within the alpha-shape of its corresponding $L C S_{i, j}$ of the $\mathrm{AH}$.

(3) Computation of an index for each digit $i$ as the average of the fraction $n_{i, j} / n_{p}$, across all the LCSs of that digit.

$$
i_{i}=\frac{1}{5 \cdot n_{p}} \sum_{j=1}^{5} n_{i, j}
$$

(4) The $A I K C_{2}$ was obtained by weighting the index of each digit with the same coefficients as in Eq. (11):

$$
A I K C_{2}=\sum_{i=1}^{5} w_{i} \cdot i_{i} .
$$

Matlab was used to compute $A I K C_{2}$ with the predefined functions alphaShape, inShape. For the computation of the alpha-shape for each LCS, the alpha radius was selected using the Matlab function criticalAlpha, under the condition that the alpha-shape should be defined with only one region.

\section{3 $A I K C_{3}$ : based on the comparison of common grasping postures}

A third option proposed here to define anthropomorphism is based on comparing the positions and orientations adopted by the LCSs of the $\mathrm{AH}$ and $\mathrm{HH}$ when grasping a set of objects that are representative of typical grasping postures employed during daily life. In this study, we propose to obtain the position and orientation of the LCSs by simulation using the kinematic models.

Three objects are initially proposed: a cylinder, a sphere and a prism, representative of cylindrical, spherical, and extension grips. In order to simulate each grasp, an optimization process was undertaken, with the joint angles as variables, in order to minimize an objective function that was representative of the intended type of grasp. Fig. 3 shows the $\mathrm{KC}$ of the $\mathrm{HH}$ in the optimized grasping posture for each object.
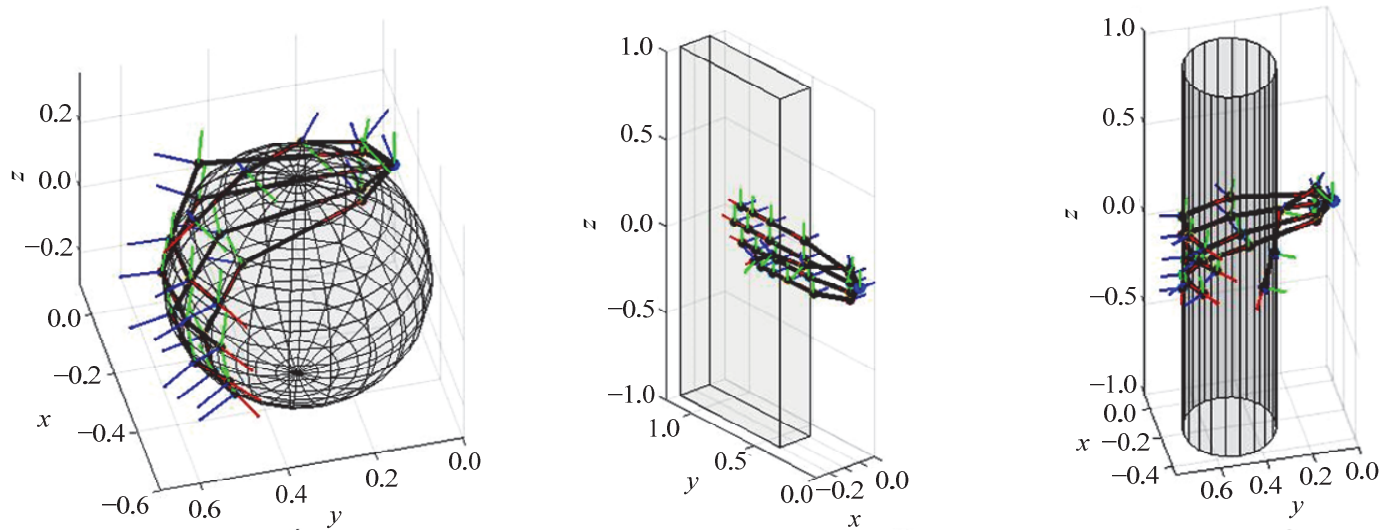

Fig. 3 Grasping postures of the human hand on the three selected objects placed in the fixed $\left\{L C S_{0}\right\}$ of the wrist. The LCS associated with each joint is shown: blue $X$-axis, red $Y$-axis, green $Z$-axis. 
The computation of $A I K C_{3}$ is performed following these steps:

(1) Simulation of the grasping postures with both $\mathrm{HH}$ and $\mathrm{AH}$ on each of the $k$ objects $(k=1$ to $k=3$ in this study). This implies the minimization of an objective function $f_{k}$ that is different for each object (see Supplementary Materials), using the angles of $\mathrm{F} / \mathrm{E}$ and $\mathrm{Ab} / \mathrm{Ad}$ of the joints, $\beta_{i j}$ and $\alpha_{i j}$, as variables.

(2) Computation of the transformation matrices of each LCS with respect to the fixed one $\left(\left\{L C S_{0}\right\}\right.$ in the wrist), in each grasping posture using Eq. (6).

(3) Computation of the AI for the posture corresponding to each object $k$ using Eq. (14). This is similar to Eq. (11), but here the translation and orientation errors are computed from the transformations of each LCS with respect to $\left\{L C S_{0}\right\}$ instead of its immediately proximal LCS in the $\mathrm{KC}$, and the error in the mobility range does not apply, because one specific posture is being compared.

$$
A I K C_{3 k}=1-\sum_{i=1}^{5} w_{i} \cdot\left[\frac{1}{n_{i}} \sum_{j=1}^{n_{i}} e_{t_{i, j}}+\frac{1}{n_{i}} \sum_{j=1}^{n_{i}} e_{o_{i, j}}\right] / 2,
$$

(4) Computation of $A I K C_{3}$ as the average across objects, namely:

$$
A I K C_{3}=\frac{1}{k} \sum_{k=1}^{k} A I K C_{3 k} .
$$

\section{Evaluation of artificial hands}

In order to evaluate and compare the usefulness and effectiveness of the indexes defined here, they were computed for various AHs, using Matlab. The AHs were (Fig. 4): (a) Barrett ${ }^{[33]}$, which is a three-finger robotic gripper; (b) BruJa ${ }^{[34]}$ and (c) IMMA ${ }^{[35]}$, both low-cost anthropomorphic prosthetic hands designed by the au- thors' research group; (d) Shadow hand, claimed to be the most anthropomorphic robotic hand ${ }^{[13]}$; and (e) $\mathrm{i}$-Limb hand ${ }^{[12]}$, one of the most advanced commercial prosthetic hands.

The data about the kinematic structure of each hand (Eq. (1)) and the mobility ranges in its joints are shown in the Supplementary Materials. HH data were obtained from previous works ${ }^{[22,25]}$, and the geometric information about the AHs was taken from the data supplied by the manufacturers or designers.

\section{Results}

Table 1 shows the AIKCs $(s=1,2,3)$ for the hands analyzed, in which value 1.0 means a perfect mimicry of the HH model. The Shadow hand is the most anthropomorphic and the Barrett hand the least anthropomorphic according to any of the indexes, although the $A I K C_{2}$ of the Barrett hand is comparable to that of the BruJa and IMMA hands. $A I K C_{1}$ and $A I K C_{3}$ results are much higher than those of $A I K C_{2}$. According to $A I K C_{2}$, the degree of anthropomorphism of all the hands is low, in the order of $15 \%$ for the Shadow hand, and in the order of $1 \%-2 \%$ for the Barrett, BruJa, IMMA, and i-Limb hands. This is because the workspace of each finger is planar under the premise of considering the absence of $\mathrm{Ab} / \mathrm{Ad} \mathrm{DoF}$ in the MCP joints of these AHs. In fact, to avoid obtaining null volumes of the alpha-shape, the simulation was performed by setting an $\mathrm{Ab} / \mathrm{Ad}$ range of $\pm 1^{\circ}$ in their $\mathrm{MCP}$ joints. This mobility could be assumed due to the flexibility of the joints themselves. For example, Fig. 5 shows the alpha-shape of the workspace associated with the LCS of every joint in the human and the IMMA hands.

The results shown in Table 1 also indicate a clear correlation between $A I K C_{1}$ and $A I K C_{3}$. The correlation coefficient between these indexes is igher than $99 \%$,

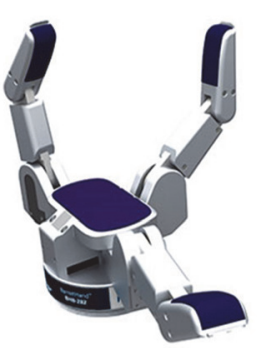

(a) Barrett (CBarrett Technology

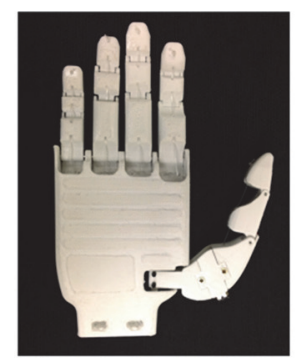

(b) BruJa

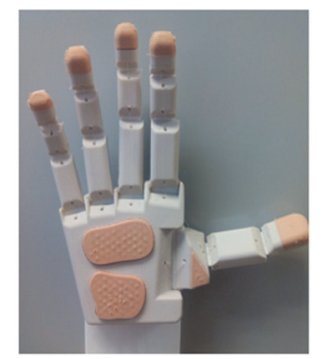

(c) IMMA

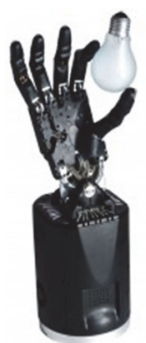

(d) Shadow CShadow Robot Company

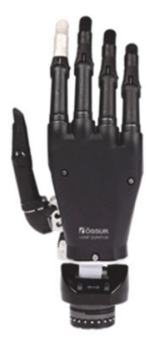

(e) i-Limb CÖssur

Fig. 4 Artificial hands analyzed and compared in this study. 
Table 1 Anthropomorphism indexes obtained for each artificial hand

\begin{tabular}{cccc}
\hline Hand & $A I K C_{1}$ & $A I K C_{2}$ & $A I K C_{3}$ \\
\hline Barrett & 0.51 & 0.01 & 0.37 \\
BruJa & 0.80 & 0.01 & 0.73 \\
IMMA & 0.84 & 0.01 & 0.78 \\
Shadow & 0.87 & 0.15 & 0.81 \\
i-Limb & 0.80 & 0.02 & 0.78 \\
\hline
\end{tabular}

despite the fact that $A I K C_{1}$ compares the kinematic structure and the mobility ranges, and $A I K C_{3}$ compares the $\mathrm{KC}$ but with the positions and orientations of the LCSs in grasping postures.

The differences in $A I K C_{3}$ across objects were small for all hands, ranging from 0.34 to 0.38 for Barrett, from 0.67 to 0.78 for BruJa, from 0.74 to 0.85 for IMMA, from 0.76 to 0.84 for Shadow, and from 0.77 to 0.81 for i-Limb.

\section{Discussion}

In this study, three indexes have been defined and compared to quantify the anthropomorphism of the $\mathrm{KC}$ of AHs. These indexes are straightforward computed and can be useful during the initial design stages of AHs, or to evaluate them, as performed in this study with five different AHs. They evaluate important aspects of anthropomorphism in accordance with previous studies $^{[16,18,19]}$, and overcome some of their limitations. Here, we considered all the parameters for defining the $\mathrm{KC}$, that is, the position and orientation of all the joints including all the finger segments. In Ref. [18], only the fingertips were considered to define the workspace of the hand and in Ref. [19] the orientation differences only were considered in the MCP joints. Moreover, the orientations are compared while computing the indexes by means of quaternions ${ }^{[28]}$, as a more accurate alternative to Euler angles ${ }^{[19]}$ or rotation matrices ${ }^{[18]}$. The use of the alpha-shape proposed here as an alternative to the convex-hull to compare the workspace avoids the inclusion of interior regions or other areas that do not strictly pertain to the reachable workspace ${ }^{[19]}$.

The low values obtained in $A I K C_{2}$ for all the AHs analyzed are explained by the much smaller workspace reachable by their finger joints as compared with the $\mathrm{HH}$ (see Fig. 5). To confirm this, we analyzed a hand with the same kinematic structure as the $\mathrm{HH}$ but with the

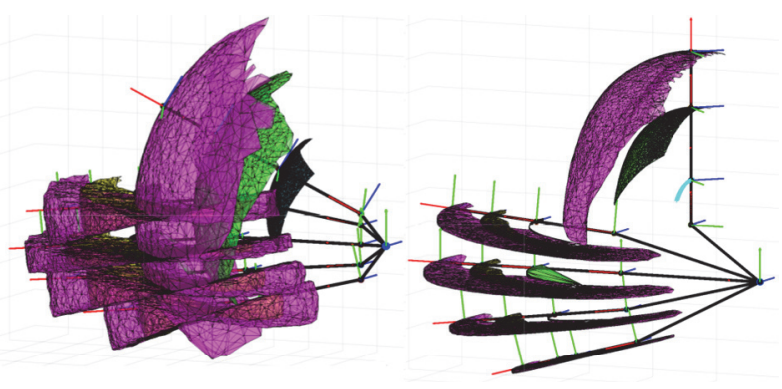

Fig. 5 Alpha-shape of the human hand (left) and IMMA hand (right) workspaces for each joint (fingertip: pink, DIP: yellow, PIP and IP: pink, MCP: green).

mobility range of $\mathrm{Ab} / \mathrm{Ad}$ in the $\mathrm{MCP}$ joints of all fingers reduced to $\pm 1^{\circ}$, resulting in an $A I K C_{2}$ of 0.07 . The combination of $\mathrm{Ab} / \mathrm{Ad}$ together with $\mathrm{F} / \mathrm{E}$ movements in the same finger is what allows the $\mathrm{HH}$ to increase the volume of its workspace. Indeed, the Shadow hand achieves a higher $A I K C_{2}$ because its MCP joints have two DoFs (F/E and $\mathrm{Ab} / \mathrm{Ad})$. In the Barrett hand, the $\mathrm{CMC}$ joint allowing $\mathrm{Ab} / \mathrm{Ad}$ in the index and middle fingers, combined with the flexion of the MCP and interphalangeal joints, compensates for the fact that the hand consists of only three fingers and achieves an $A I K C_{2}$ comparable to that of apparently more anthropomorphic hands.

Anthropomorphism estimations obtained in previous works maintain a certain correlation with those obtained in this study, despite being based on different definitions. For example, in Liarokapis et al. ${ }^{[19]}$ the total anthropomorphism score was around $10 \%$ for Barrett and close to $40 \%$ for Shadow. These values are higher than those obtained in $A I K C_{2}$ because they are based on the comparison of workspace volumes using convex-hulls instead of alpha-shapes. Our results are not directly comparable to those of Feix et al. ${ }^{[18]}$, since different hands were analyzed, although the orders of magnitude were similar to those obtained here with $A I K C_{2}$. In Ref. [18], a value of $5.2 \%-9.2 \%$ (depending on the sampling method) was obtained for the hand with the highest number of DoFs analyzed (FRH-4) and $0.25 \%$ for the prosthetic hand SensorHand ${ }^{\odot}$ (Otto Bock) with three fingers, which is comparable to the Barrett hand. In Ref. [17], the results obtained for the AIM were: $27 \%$ Barrett, 42\% IMMA, 45\% i-Limb, and $88 \%$ Shadow. The results are comparable to those of the present study, in the sense that in both studies the Shadow 
and the Barrett hands are the most and the least anthropomorphic, respectively. However, $A I K C_{1}$ and AIM rank the IMMA and i-Limb differently. Furthermore, the Shadow hand exhibits higher AIM values (almost double) compared to the previous ones, while for the $A I K C_{1}$ and $A I K C_{3}$ these differences are lower than $10 \%$. The differences between the results of the two studies may be caused by the fact that the number of actuators and type of underactuation, considered in the AIM definition, were not taken into account in this study.

The high correlation obtained between $A I K C_{1}$ and $A I K C_{3}$ is unexpected. Although both indexes are calculated in a similar way, $A I K C_{3}$ does not include information about the mobility ranges, but only about the positions and orientations of the LCSs, with a different reference and in different postures. The correlation between the two indexes would probably decrease if the number of grasping postures increases. This point should be investigated in future works. If the level of correlation between the indexes remains high after increasing the grasping postures, this would suggest that the information they provide is redundant and therefore the use of $A I K C_{1}$ would be preferable since its calculation is more straightforward. In contrast, the information provided by indexes $A I K C_{1}$ and $A I K C_{2}$ is complementary, since hands with different kinematic structures, with a different number of fingers and/or phalanges, and therefore very different in terms of the parameters of the $\mathrm{KC}$, such as the Barrett and IMMA or BruJa hands, obtain a similar degree of anthropomorphism in terms of workspace.

The AIs defined in this study could help to define optimal solutions that maximize the degree of anthropomorphism. Nevertheless, this study has some limitations. The definition of the AIKCs is based on a comparison with a kinematic model of the $\mathrm{HH}$ that is not perfect because, although it considers all its main DoFs, it assumes universal joint models in the joints with 2 DoFs, which is questionable ${ }^{[21,36]}$. Moreover, the assumption that a single kinematic model of the $\mathrm{HH}$ allows representation of the hands of different subjects seems to be dubious ${ }^{[21]}$. In any case, the model considered is representative of the most advanced and recent biomechanical models of the $\mathrm{HH}^{[22,23,25]}$. Even if new improved hand models are developed in the near future, it would always be possible to maintain, with minor changes, the way in which our indexes are calculated. The mobility range of the different joints of the $\mathrm{HH}$ used here could also be revised in the future without major changes in the general methodology. Despite the fact that some recent works have analyzed this last point, they are partial and with little agreement among them ${ }^{[37,38]}$. In addition, $A I K C_{2}$ and $A I K C_{3}$ could be improved with experimental data measured on the $\mathrm{HH}$, as in Ref. [18], to obtain a more realistic comparison. Finally, another limitation of the approach followed here is that synergies between the actuation of the various DoFs are not considered in the $\mathrm{HH}$ or in the $\mathrm{AH}$. In fact, in the $\mathrm{HH}$, the mobility ranges of the joints are variable depending on the posture, and in the AHs, which are usually underactuated, each actuator moves more than one DoF, thus affecting the mobility of the different joints. In this sense, we can say that the AIs defined in this study only consider the structure of the $\mathrm{KC}$, but not its relation with the method of actuation. Including the actuation synergies would allow more complete AIs to be defined and is a further development of this study.

\section{Conclusion}

Three indexes for the definition of the degree of anthropomorphism of the $\mathrm{KC}$ of an $\mathrm{AH}$ have been defined and compared. As a first conclusion, $A I K C_{1}$, based on the comparison of the defining parameters of the $\mathrm{KC}$ (position and orientation vectors, and mobility ranges) and $A I K C_{3}$, based on the comparison of the postures performed during the grasping of characteristic objects, show a high correlation for the hands compared, with the degree of anthropomorphism being around $70 \%-90 \%$ for the five-finger hands and around $40 \%-50 \%$ for the three-finger Barrett hand. As a second conclusion, the AI based on the comparison of the workspace reachable by the joints of the $\mathrm{KC}, A I K C_{2}$, presents much lower values than the previous ones (ranging between $1 \%$ and $15 \%$ ), and are higher for hands that, like Shadow, combine $\mathrm{Ab} / \mathrm{Ad}$ and $\mathrm{F} / \mathrm{E}$ movements in the $\mathrm{KC}$ of each finger. As a final conclusion, among the hands compared, the Shadow robotic hand is the one that overall presents the highest values with the defined indexes. The advanced commercial prosthesis i-Limb presents AIKCs comparable to other low-cost $A H s$. The AIKCS for the three-finger Barrett robotic hand are lower, although the $A I K C_{2}$ value 
is comparable to that of other five-finger hands.

\section{Acknowledgment}

This work was supported by the Spanish Ministry of Economy and Competitiveness and ESF (Grant No. BES-2015-076005); the Spanish Ministry of Economy and Competitiveness, AEI and ERDF (Grant Nos. DPI2014-60635-R and DPI2017-89910-R); and Universitat Jaume I, Spain (Grant No. UJI-B2017-70).

Open Access This article is licensed under a Creative Commons Attribution 4.0 International License, which permits use, sharing, adaptation, distribution and reproduction in any medium or format, as long as you give appropriate credit to the original author(s) and the source, provide a link to the Creative Commons licence, and indicate if changes were made.

The images or other third party material in this article are included in the article's Creative Commons licence, unless indicated otherwise in a credit line to the material. If material is not included in the article's Creative Commons licence and your intended use is not permitted by statutory regulation or exceeds the permitted use, you will need to obtain permission directly from the copyright holder.

* All supplementary materials are available at http://www.springer.com/journal/42235.

\section{References}

[1] Parada Puig J E, Rodriguez N E N, Ceccarelli M. A methodology for the design of robotic hands with multiple fingers. International Journal of Advanced Robotic Systems, 2008, 5, 177-184.

[2] Kemp C, Edsinger A, Torres-Jara E. Challenges for robot manipulation in human environments [grand challenges of robotics]. IEEE Robotics \& Automation Magazine, 2007, 14, 20-29.

[3] Liarokapis M V, Artemiadis P K, Kyriakopoulos K J. Functional anthropomorphism for human to robot motion mapping. IEEE International Workshop on Robot and Human Interactive Communication, Paris, France, 2012, 31-36.

[4] Belter J T, Segil J L, Dollar A M, Weir R F. Mechanical design and performance specifications of anthropomorphic prosthetic hands: A review. The Journal of Rehabilitation Research and Development, 2013, 50, 599-618.

[5] ten Kate J, Smit G, Breedveld P. 3D-printed upper limb prostheses: A review. Disability and Rehabilitation: Assis- tive Technology, 2017, 12, 300-314.

[6] Gibbard J. Open Hand Project, [2018-03-01], www.openhandproject.org

[7] Gibbard J, Payne S, Open Bionics, [2018-03-01], www.openbionics.com

[8] Duong T, Wagner B, Abraham T, Davidson M, Bains G, Daher N, Friedrich A. Comparative study of functional grasp and efficiency between a 3D-printed and commercial myoelectric transradial prosthesis using able-bodied subjects: A pilot study. Journal of Prosthetics and Orthotics, 2017, 29, 112-118.

[9] Andrychowicz M, Baker B, Chociej M, Józefowicz R, McGrew B, Pachocki J, Petron A, Plappert M, Powell G, Ray A, Schneider J, Sidor S, Tobin J, Welinder P, Weng L, Zaremba W. Learning dexterous in-hand manipulation. International Journal of Robotics Research, 2019, 39, 3-20.

[10] Li S, Ma X J, Liang H Z, Gorner M, Ruppel P, Fang B, Sun F $\mathrm{C}$, Zhang J W. Vision-based teleoperation of shadow dexterous hand using end-to-end deep neural network. International Conference on Robotics and Automation (ICRA), Montreal, Canada, 2019, 416-422.

[11] Simon A M, Turner K L, Miller L A, Hargrove L J, Kuiken T A. Pattern recognition and direct control home use of a multi-articulating hand prosthesis. IEEE International Conference on Rehabilitation Robotics, Toronto, Canada, 2019, 386-391.

[12] ÖSSUR. I-Limb, [2019-12-01], https://www.ossur.com/en-us/ prosthetics/arms/i-limb-ultra

[13] Greenhill R, Elias H. Shadow Dexterous Hand, [2018-03-01], https://www.shadowrobot.com/wp-content/ uploads/shadow_dexterous_hand_technical_specification_E _20150827.pdf

[14] Craig J J. Introduction to Robotics: Mechanics and Control, 3rd, Prentice Hall, 2004, 1, 408.

[15] Biagiotti L, Lotti F, Melchiorri C, Vassura G. How Far is the Human Hand? A Review on Anthropomorphic Robotic Endeffectors, University of Bologna, Internal Report, Italy, 2004.

[16] Jiang L, Liu Y, Yang D P, Liu H. A synthetic framework for evaluating and designing an anthropomorphic prosthetic hand. Journal of Bionic Engineering, 2018, 15, 69-82.

[17] Llop-Harillo I, Pérez-González A, Gracia-Ibáñez V. Anthropomorphism index of mobility for artificial hands. Applied Bionics and Biomechanics, 2019, 2019, 7169034.

[18] Feix T, Romero J, Ek C H, Schmiedmayer H B, Kragic D. A metric for comparing the anthropomorphic motion capability of artificial hands. IEEE Transactions on Robotics, 2013, 29, 82-93. 
[19] Liarokapis M V, Artemiadis P K, Kyriakopoulos K J. Quantifying anthropomorphism of robot hands. IEEE International Conference on Robotics and Automation, Karlsruhe, Germany, 2013, 2041-2046.

[20] Kapandji I A. The Physiology of the Joints: Upper Limb, Churchill Livingstone, New York, USA, 1982.

[21] Santos V J, Valero-Cuevas F J. Reported anatomical variability naturally leads to multimodal distributions of denavithartenberg parameters for the human thumb. IEEE Transactions on Biomedical Engineering, 2006, 53, 155-163.

[22] Sancho-Bru J L. Model Biomecànic de La Mà Orientat Al Disseny D'eines Manuals, PhD Thesis, Universitat Jaume I, Castelló, Spain 2000. (in Valencian)

[23] Peña-Pitarch E, Falguera N T, Yang J. Virtual human hand: Model and kinematics. Computer Methods in Biomechanics and Biomedical Engineering, 2014, 17, 568-579.

[24] Cobos S, Ferre M, Sanchez Uran M A, Ortego J, Pena C. Efficient human hand kinematics for manipulation tasks. IEEE/RSJ International Conference on Intelligent Robots and Systems, Nice, France, 2008, 2246-2251.

[25] León B, Morales A, Sancho-Bru J. From Robot to Human Grasping Simulation, Springer, Cham, 2014.

[26] Hartenberg R S, Denavit J. A kinematic notation for lowerpair mechanisms based on matrices. Transactions of the ASME - Journal of Applied Mechanics, 1955, 22, 215-221.

[27] Wu G, Cavanagh P R. ISB recommendations for standardization in the reporting of kinematic data. Journal of Biomechanics, 1995, 28, 1257-1261.

[28] Huynh D Q. Metrics for 3D rotations: Comparison and analysis. Journal of Mathematical Imaging and Vision, 2009, 35, 155-164.

[29] Soucacos P N. Indications and selection for digital amputation and replantation. Journal of Hand Surgery, 2001, 26 $572-581$.
[30] Dermitzakis K, Ioannides A, Lin H T. Robotic thumb grasp-based range of motion optimization. 35th Annual International Conference of the IEEE Engineering in Medicine and Biology Society (EMBC), 2013, 3163-3166.

[31] Barber C B, Dobkin D P, Huhdanpaa H. The quickhull algorithm for convex hulls. ACM Transactions on Mathematical Software, 1996, 22, 469-483.

[32] Edelsbrunner H. Alpha shapes - A survey. Tessellations in the Sciences, 2010, 1-25.

[33] Townsend W. Barrett Hand, [2018-03-01], https://advanced. barrett.com/barretthand.

[34] Andrés F J, Pérez-González A, Rubert C, Fuentes J, Sospedra B. Comparison of grasping performance of tendon and linkage transmission systems in an electric-powered low-cost hand prosthesis. Journal of Mechanisms and Robotics, 2019, 11, 011018.

[35] Llop-Harillo I, Pérez-González A. System for the experimental evaluation of anthropomorphic hands. Application to a new 3D-printed prosthetic hand prototype. International Biomechanics, 2017, 4, 50-59.

[36] Stillfried G, Hillenbrand U, Settles M, van der Smagt P. MRI-Based skeletal hand movement model. In: Balasubramanian R, Santos V J, eds., The Human Hand as an Inspiration for Robot Hand Development, Springer International Publishing, 2014, 49-75.

[37] Coupier J, Hamoudi S, Telese-Izzi S, Feipel V, Rooze M, Van Sint Jan S. A novel method for in-vivo evaluation of finger kinematics including definition of healthy motion patterns. Clinical Biomechanics, 2016, 31, 47-58.

[38] Gracia-Ibáñez V, Vergara M, Sancho-Bru J L, Mora M C, Piqueras C. Functional range of motion of the hand joints in activities of the international classification of functioning, disability and health. Journal of Hand Therapy, 2017, 30, $337-347$.

\section{Appendix}

The spreadsheet file "DataKinematicChain.xlsx" in the Supplementary file 1 contains, for the HH and each AH analyzed in this study, the elements of the vectors $v_{i, j}$ in Eq. (1) that define their KCs. For each segment, the vector of its LCS with respect to its immediate proximal LCS in the chain is shown. Note that $x, y, z$ are non-dimensional values normalized to the hand length (distance from the wrist to the fingertip of the middle finger at the RP), and $\theta_{x}, \theta_{y}, \theta_{z}$ are the Euler angles around the $X, Y$ and $Z$ axes respectively, with order of rotations $X Z Y$, expressed in radians. For the digits with four segments, the row labeled medial phalanx actually corresponds to the distal one and the row labeled distal phalanx corresponds to the fingertip of the digit. The spreadsheet also contains the minimum and maximum range of mobility of each joint around the reference posture for the $\mathrm{HH}$ and each $\mathrm{AH}$ analyzed.

The document "GraspingPostures.pdf" in the Supplementary file 2 contains the optimization functions $f_{k}$ used to obtain the grasping postures of the different objects and all their geometrical dimensions and their positions for computing $\mathrm{AIKC}_{3}$. 